\title{
THE QUEST FOR AUTOMATED LAND COVER CHANGE DETECTION USING SATELLITE TIME SERIES DATA
}

\author{
${ }^{\dagger}$ B.P. Salmon, ${ }^{\dagger *}$ J.C. Olivier, ${ }^{\dagger} W$ W. Kleynhans, ${ }^{\ddagger}$ K.J. Wessels and ${ }^{\ddagger} F$. van den Bergh \\ ${ }^{\dagger}$ Department of Electrical, \\ Electronic and Computer \\ Engineering, University of \\ Pretoria, South Africa \\ ${ }^{*}$ Defense, Peace, Safety and \\ Security Unit, CSIR, Pretoria, \\ South Africa
}

\begin{abstract}
This paper shows that a feedforward Multilayer Perceptron (MLP) operating over a temporal sliding window of multispectral time series MODerate-resolution Imaging Spectroradiometer (MODIS) satellite data is able to detect land cover change that was artificially introduced by concatenating time series belonging to different types of land cover. The method employs an iteratively retrained MLP that is a supervised method, and thus captures all local environmental patterns. Depending on the length of the temporal sliding window used in the short-term Fourier transform, an overall change detection accuracy of between $87.62 \%$ and $97.02 \%$ was achieved. It is shown that for this type of simulated land cover change, where land cover change was abrupt, a short-term FFT window of 18 months or less, using only the two NDVI spectral bands of MODIS data was sufficient to detect change reliably.
\end{abstract}

Index Terms - Classification, feedforward neural networks, satellites, time series

\section{INTRODUCTION}

Automation of monitoring land cover change at regional or global scales using hyper-temporal multi-spectral coarse resolution satellite data has been a highly desired, but often elusive goal of environmental remote sensing [1]. Land cover change often indicates land use change with major socioeconomic impacts, while the transformation of vegetation cover (e.g. deforestation, agricultural expansion, urbanisation) have signicant impacts on hydrology, ecosystems and climate [2, 3]. Digital change detection encompasses the quantification of temporal phenomena from multi-date imagery that is most commonly acquired by satellite-based multi-spectral sensors [4]. For regional applications, these methods need to be sufficiently automated when processing exceptionally large volumes of data [5]. As global datasets become more accessible and computational resources become

This research was funded by the CSIR Strategic Research Panel. more affordable, such global or regional automated change detection systems should become more attainable. Due to the complex and non-parametric nature of land cover classication and change detection, machine learning methods are widely regarded as the most viable option for automated change detection [5].

This paper presents a different approach towards achieving the goal of automated land cover change detection. The method uses a supervised MLP that incorporates a sliding window that capitalizes on the high temporal sample rate of coarse resolution satellite data. This allows the observation of seasonal land surface reflectance patterns which facilitates distinguishing different land cover types and provides a signal for applying advanced time series analysis [6]. The Multilayer Perceptron (MLP) has been shown to be able to learn the complex interdependecies of the multi-spectral time series and was thus used for land cover classification rather than maximum likelihood (ML) detection [7].

The paper is organized as follows. Section 2 will present the key ideas behind the MLP, including the use of the temporal sliding window and short term Fast Fourier Transform. Section 3 presents the results for the automated change detection on simulated land cover change. Section 4 presents the conclusions.

\section{CHANGE DETECTION USING A SUPERVISED MLP AND A SLIDING WINDOW FFT}

A supervised MLP was used for change detection and thus the classifier requires training data. A training set must be defined for each study area to ensure that the classifier captures local patterns. Study areas were selected where settlements and natural vegetation occur in close proximity to ensure that the rainfall, soil type and local climate were similar over both landcover types. The study areas were: 1) Koelemansrus (28.85E, 24.23S) - 17km south-west of Makopane and 2) Seshego (29.40E, 23.81S) - 10km north-west of Polokwane. Training areas were selected using visual interpretation 
Table 1. Number of pixels per land cover type, per study area for training, validation and testing sets.

\begin{tabular}{|l|c|c|c|}
\hline Study Area & Training Time Series & Validation Time Series & Simulated Change Time Series \\
\hline Area 1 - Natural & 225 & 225 & 84 \\
Area 1 - Settlement & 82 & 82 & 84 \\
Area 2 - Natural & 42 & 43 & 42 \\
Area 2 - Settlement & 162 & 162 & 42 \\
\hline Total - Natural & 267 & 268 & 126 \\
Total - Settlement & 244 & 244 & 126 \\
\hline
\end{tabular}

of SPOT5 high resolution images (2006) to ensure that none of the time series experienced any land cover change during the study period. The number of time series selected for each class, per study area, is given in Table 1 . These training sets represent the two valid classes for the MLP in training mode. The MLP also relies on independent (not included in training phase) validation sets to verify the perceptron's generalization [8]. The separation between the training set and validation set was done using a pseudorandom sequence to partition the time series.

Since information on known land cover change is scarce and land cover change is a rare event within a regional landscape [4], the land cover change was simulated, which made it possible to control both the type, rate and timing of change in order to evaluate the change detection methods. The type, rate and timing of the land cover change are functions of anthropogenic activities and environmental conditions, and could be encompassed within a model where these parameters are known. However, since examples of change are so poorly documented and mapped, it is very hard to feasibly train a supervised MLP on examples of change, or to model change by introducing all permutations of the afore-mentioned parameters.

Land cover change was simulated by concatenating the time series for a pixel from a natural vegetation class (class 1) to that of a pixel from a human settlement (often informal and unplanned) (class 2). Control sets containing no change were also created by concatenating a set of time series from a natural vegetation class (settlement class) to another set of time series also from a natural vegetation class (settlement class ). Hence there were four testing data subsets based on concatenating time series of different combination of time series:

- subset 1: natural vegetation time series (class 1) spliced to settlement time series (class 2);

- subset 2: settlement time series (class 2) spliced to natural vegetation time series (class 1);

- subset 3: settlement time series (class 2) spliced to another settlement time series (class 2);

- subset 4: natural vegetation time series (class 1) spliced to another natural vegetation time series (class 1).
These four subsets were used to produce a confusion matrix to test if the operational MLP can detect change reliably in an automated fashion on subsets 1 and 2, while not falsely detecting change for subsets 3 and 4 . This particular splicing method produced an abrupt change, which does not necessarily represent the reality of human-induced change such as settlement expansion, that may take several months to develop. The notion of a blending period (linear blend over 12 and 24 months) from one land cover time series to another was initially considered. The blending model does not reflect all models of actual land cover change but it does illustrate that the blending period does not affect the ability to correctly detect change but merely delays the period before the MLP stabilizes into the correct class.

The MLP was required to process time series data and detect land cover change as a function of time. This requires a temporal sliding window that operates on a subsequence of the time series for the two NDVI spectral bands (Figure 1). The sliding window has a length $Q$ of 6,12 and 18 months and was moved at 8-day increments through the time series. Since the delay in change detection in the initial training period was determined by the length of the window $Q$, the effect of its length on the accuracy of automated change detection was

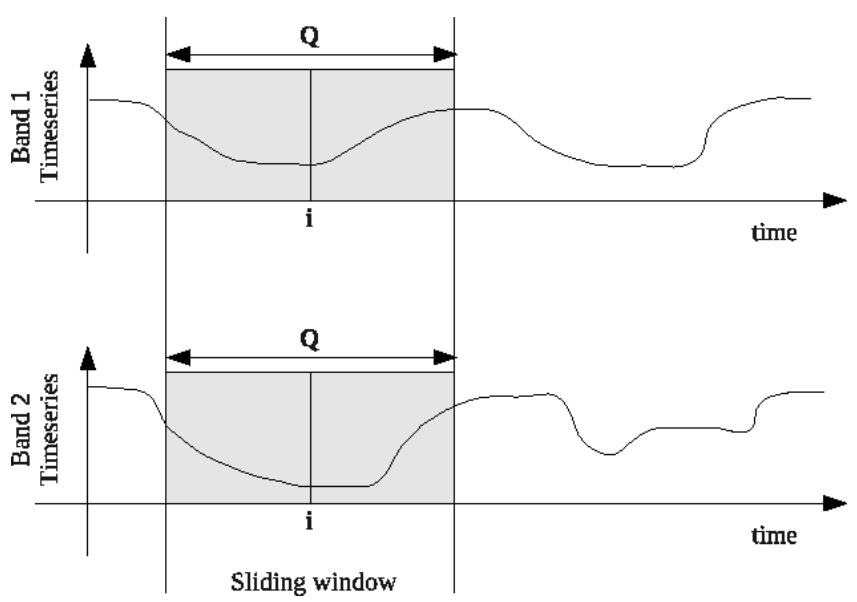

Fig. 1. Temporal sliding window used to define a subsequence of the time series for classication and change detection. 
Table 2. Classification accuracy of the iteratively trained MLP on the validation set. The table reports on the average classification accuracy and standard deviation for each of the classes for temporal sliding windows of various lengths.

\begin{tabular}{|c|c|c|c|}
\hline Area & \multicolumn{3}{|c|}{ Study Area 1 : Koelemansrus $(28.85 \mathrm{E}, 24.23 \mathrm{~S})$} \\
\hline Sliding window length & 6 months & 12 months & 18 months \\
\hline Vegetation validation & $\begin{array}{c}96.76 \% \\
(2.35)\end{array}$ & $\begin{array}{c}97.04 \% \\
(2.17)\end{array}$ & $\begin{array}{c}97.73 \% \\
(1.96)\end{array}$ \\
\hline Settlement validation & $\begin{array}{c}88.24 \% \\
(5.28)\end{array}$ & $\begin{array}{c}90.70 \% \\
(3.39)\end{array}$ & $\begin{array}{c}91.18 \% \\
(3.15)\end{array}$ \\
\hline Overall validation & $94.48 \%$ & $95.35 \%$ & $95.98 \%$ \\
\hline Area & \multicolumn{3}{|c|}{ "Study Area 2 : Seshego (29.40E, 23.81S) } \\
\hline Sliding window & 6 months & 12 months & 18 months \\
\hline Vegetation validation & $\begin{array}{l}75.16 \% \\
(11.08)\end{array}$ & $\begin{array}{c}81.98 \% \\
(6.74)\end{array}$ & $\begin{array}{c}84.79 \% \\
(6.15)\end{array}$ \\
\hline Settlement validation & $\begin{array}{c}96.67 \% \\
(2.15)\end{array}$ & $\begin{array}{c}97.22 \% \\
(2.14)\end{array}$ & $\begin{array}{c}97.74 \% \\
(1.85)\end{array}$ \\
\hline Overall validation & $92.16 \%$ & $94.02 \%$ & $95.02 \%$ \\
\hline
\end{tabular}

studied. Longer sliding windows spanning multiple months were expected to compensate for the effects of seasonal climate variability, but at the cost of delayed change detection.

The Fast Fourier Transform (FFT) [9] was computed over the $Q$-length time sequence to produce the frequency spectrum. The first 20 components were the dominant components in the spectrum (10 dominant components for $Q=$ 6 months) and were extracted from the two NDVI spectral bands to represent the feature vector inputs to the MLP. Note that in this paper change detection was only performed on a per pixel basis, and contextual information in relation to neighboring time series was not included.

The MLP employed comprises an input layer, one hidden layer and an output layer. The hidden and output layer both used a tangent sigmoid activation function in each node. The input layer accepts input vectors for classification, while the output layer represents the likelihood that an input belongs to a specific class. For example, in the two-class case presented in this paper, the MLP output is in the range $(-1 ; 1)$, where 1 represents $100 \%$ certainty of the input belonging to class 1 (natural vegetation), while a -1 represents $100 \%$ certainty of class 2 (settlement). An output value of 0 indicates the highest level of uncertainty of class membership and was thus assigned as the decision threshold. The weights in the training phase of the MLP were determined using a steepest descent gradient optimization method, with gradients estimated using backpropagation [8]. The validation set was used for initial MLP architecture optimization by testing the generalization error to identify overfitting of the network for each study area. A moving average window length of 3 was also applied to the MLP outputs to smooth out all transitory oscillations in class labels caused by higher uncertainty in the classification.

\section{EXPERIMENTAL RESULTS}

At a given time $i$ the MLP is trained to recognize data from the training set at time $i$ in the time series, and will be used to classify the data from the testing set at time $i$. Because of the retraining at each time increment the adaptation of the weights was fast and of low complexity, as there were only small MLP weight changes over each 8 day increment.

The classification accuracy results on the validation sets for sliding window sizes of 6,12 and 18 months using the NDVI spectral bands of MODIS are provided in Table 2. Each entry in Table 2 gives the average classification accuracy for each area, calculated over 10 independent repeated experiments. The standard deviation for each scenario is given in parentheses. An overall improvement in classification accuracy was observed when the sliding window length $Q$ was increased to 18 months.

The outcome of the change detection simulations for both study areas is summarised in the confusion matrix (Table 3 ). The land cover change detection accuracy improves by almost $5 \%$ when the length of the sliding window $Q$ was increased. The drawback was that more time series acquistions were needed for this improvement and thus a longer delay was experienced in change detection. From all the results presented it is clear that change detection can be ensured when using the 2 NDVI spectral bands of the MODIS sensor.

\section{CONCLUSION}

This study demonstrated that a MLP operating on a temporal sliding window of MODIS time series data was able to detect simulated land cover change in an automated fashion after initial training. Experimental results indicated that the MLP could detect change accurately at rates of higher than $87.62 \%$ 
Table 3. Confusion matrix representing the land cover change detection accuracy for the study areas for temporal sliding windows of various lengths.

\begin{tabular}{|c|c|c|c|}
\hline Area & \multicolumn{3}{|c|}{ Study Area 1 : Koelemansrus $(28.85 \mathrm{E}, 24.23 \mathrm{~S})$} \\
\hline Sliding window length & 6 months & 12 months & 18 months \\
\hline $\begin{array}{l}\text { Change detected } \\
\text { (subset } 1 \& 2 \text { ) }\end{array}$ & $87.62 \%$ & $90.84 \%$ & $92.32 \%$ \\
\hline $\begin{array}{l}\text { No change detected } \\
\text { (subset } 3 \& 4 \text { ) }\end{array}$ & $87.56 \%$ & $90.78 \%$ & $92.50 \%$ \\
\hline Area & \multicolumn{3}{|c|}{ Study Area 2 : Seshego (29.40E, 23.81S) } \\
\hline Sliding window length & 6 months & 12 months & 18 months \\
\hline $\begin{array}{l}\text { Change detected } \\
\text { (subset } 1 \& 2 \text { ) }\end{array}$ & $92.14 \%$ & $96.91 \%$ & $97.02 \%$ \\
\hline $\begin{array}{l}\text { No change detected } \\
\text { (subset } 3 \& 4 \text { ) }\end{array}$ & $92.14 \%$ & $96.91 \%$ & $95.71 \%$ \\
\hline
\end{tabular}

for sliding window lengths of 6 months or longer (Table 3). The temporal sliding window on which the iteratively trained MLP operates has the advantage of continuously learning the most recent spectral properties of land cover types, as time progresses. The iteratively retrained MLP can be optimised to ensure it takes cognisance of short-term inter-annual climate variability and adapts to longer-term trends in climate without confusing any of these with land cover change, which has often been a problem with other regional land cover change studies [10]. The temporal sliding window approach can also be applied in combination with a variety of other classifiers.

Like most other supervised machine learning methods, the method proposed here is (i) dependent on periodic training data for redefining the training sets over time and (ii) has to be robust to land cover changes and other errors occurring within these training sets [5]. The main operational challenge will be the automatic generation of training data from the ancillary land cover data which is representative of the environmental diversity and diverse land uses contained within such an extensive area.

\section{REFERENCES}

[1] J. R. G. Townshend and C. O. Justice, "Selecting the spatial resolution of satellite sensors required for global monitoring of land transformations," International Journal of Remote Sensing, vol. 9, no. 2, pp. 187-236, February 1988 .

[2] R. S. DeFries, L. Bounoua, and G. J. Collatz, "Human modification of the landscape and surface climate in the next fifty years," Global Change Biology, vol. 8, no. 5, pp. 438-458, May 2002.

[3] J. A. Foley et al., "Global consequences of land use," Science, vol. 309, no. 5734, pp. 570-574, July 2005.
[4] R. S. Lunetta et al., "Land-cover change detection using multi-temporal MODIS NDVI data," Remote Sensing of Environment, vol. 105, no. 2, pp. 142-154, November 2006.

[5] R. S. DeFries and J. C. W. Chan, "Multiple criteria for evaluating machine learning algorithms for land cover classification from satellite data," Remote Sensing of Environment, vol. 74, no. 3, pp. 503-515, December 2000.

[6] J. D. Hamilton, Time Series Analysis, Princeton University Press, first edition, 1994.

[7] J. D. Paola and R. A. Schowengerdt, "A detailed comparison of back-propagation neural network and maximum likelihood classifiers for urban land use classification," IEEE Transactions on Geosience and Remote Sensing, vol. 33, no. 4, pp. 981-996, July 1995.

[8] C.M. Bishop, Neural networks for pattern recognition, Oxford University Press, New York, USA, first edition, 1996.

[9] A.V. Oppenheim, R.W. Schafer, and J.R. Buck, Discrete-Time Signal Processing, Prentice-Hall Signal Processing series, New Jersey, USA, second edition, 1999.

[10] V. Vanacker et al., "Impact of short-term rainfall fluctuation on inter-annual land cover change in sub-Saharan Africa," Global Ecology and Biogeography, vol. 14, no. 2, pp. 123-135, January 2005. 